\title{
Straight-line stabilization
}

\author{
Jian-min Mao, ${ }^{1}$ Liu Zengrong, ${ }^{2}$ and Yang Ling $^{2}$ \\ ${ }^{1}$ Department of Mathematics, Hong Kong University of Science and Technology, Hong Kong \\ ${ }^{2}$ Department of Mathematics, Shanghai University, Shanghai 201800, China
}

(Received 13 January 2000; revised manuscript received 12 May 2000)

\begin{abstract}
For finite-dimensional maps, an unstable orbit in a neighborhood of an unstable fixed point can be stabilized by adjusting parameters so that the orbit goes to the fixed point along the straight line connecting the orbit (at a given time) and the fixed point [Yang Ling, Liu Zengrong and Jian-min Mao, Phys. Rev. Lett. 84, 67 (2000)]. This is called straight-line stabilization. In this paper, we derive the expression for the region of stabilization, i.e., the region within which the straight-line stabilization method is valid. For two-dimensional maps, the parameter adjustments needed by the stabilization method are explicitly given for nine cases. Stabilization of unstable flows, with or without introducing a Poincaré map, is also investigated.
\end{abstract}

PACS number(s): 05.45.Gg, 05.45.Pq

\section{INTRODUCTION}

Chaos and instability appear, but are sometimes undesirable, in many practical problems. An unstable orbit can be guided into a stable manifold (if such a manifold exists) and is therefore stabilized, as suggested by Ott, Grebogi, and Yorke [1]. After their work, various methods for stabilization or chaos control have been proposed [2-5], and many applications of chaos control in different disciplines have been found $[6-8]$.

The idea of the straight-line stabilization method, as given in Ref. [5], is to guide an unstable orbit near an unstable fixed point to go to the fixed point directly along the straight line connecting the orbit (at a given time) and the fixed point. In this article, we further derive the expression for the size of the stabilization region (i.e., the region where the stabilization method is valid). The knowledge about the stabilization region is important, and it is particularly so if the stabilization is needed only in a certain region in phase space and, outside the region, disturbance to the original system by the stabilization process is required to be as small as possible. We also provide a means for controlling the size of the stabilization region, which may be needed in practical problems. Furthermore, for two-dimensional maps, explicit expressions of the parameter adjustments needed by the straight-line stabilization are given for nine cases. Stabilization of unstable flows with or without introducing a Poincaré map is also investigated by using the idea of the straight-line stabilization method.

This paper is organized as follows. The derivation of the expression for the stabilization region is presented in Sec. II. The nine cases for the straight-line stabilization in twodimensional maps are listed in Sec. III. The flow stabilization is investigated in Sec. IV. Conclusions are drawn in Sec. V.

\section{REGION OF STABILIZATION}

We formulate the system to be controlled by map $T: \xi_{n}$ $\rightarrow \xi_{n+1}$, where

$$
\xi_{n+1}=\mathbf{F}_{\boldsymbol{\epsilon}}\left(\xi_{n}\right)
$$

Here, $\xi \in R^{N}$ is the dynamical variable ( $N$ being a finite integer) and $\mathbf{F}_{\boldsymbol{\epsilon}}\left(\xi_{n}\right)$ is the mapping function with parameter $\boldsymbol{\epsilon}$ $\in R^{N}$. Let $\xi_{*}$ be a fixed point of the map with a nonzero $\epsilon$ and $\xi_{*}^{\mathbf{0}}$ be an unstable fixed point of the map with $\boldsymbol{\epsilon}=\mathbf{0}$. Without loss of generality, we assume that proper coordinate changes have been made so that $\xi_{*}^{0}$ is located at the origin. The straight-line stabilization method requires

$$
\xi_{n+1}=k \xi_{n},
$$

where $k$ is a real constant with absolute value less than one.

To determine the region of stabilization, we first expand each component of $\xi_{n+1}$ as a Taylor series about $\xi_{n}=\xi_{*}$, i.e.,

$$
\xi_{n+1}-\xi_{*}=\widetilde{\mathbf{J}}\left(\xi_{\mathbf{n}}-\xi_{*}\right)+\boldsymbol{\Delta}_{1}\left(\left|\xi_{n}-\xi_{*}\right|^{2}\right),
$$

where $\widetilde{\mathbf{J}}=\left(\partial \mathbf{F}_{\epsilon} / \partial \xi_{n}\right)_{\xi_{n}=\xi_{*}}$ is the Jacobian matrix of the map evaluated at $\xi_{n}=\xi_{*}$, and vector $\Delta_{1}\left(\left|\xi_{n}-\xi_{*}\right|^{2}\right)$ is the remainder in the expansion, whose leading term is of the second order in $\left|\xi_{n}-\xi_{*}\right|$. Matrix $\widetilde{\mathbf{J}}$ can be approximated by the Jacobian matrix of the map evaluated at $\xi_{*}^{0}, \quad \mathbf{J}$ $=\left(\partial \mathbf{F}_{\epsilon} / \partial \xi_{n}\right)_{\xi_{n}=\xi_{*}^{0}}$. Therefore Eq. (3) becomes

$$
\xi_{n+1}-\xi_{*}=\mathbf{J}\left(\xi_{n}-\xi_{*}\right)+\Delta_{1}\left(\left|\xi_{n}-\xi_{*}\right|^{2}\right)+\Delta_{2}\left(\left|\xi_{n}-\xi_{*}\right|^{2}\right),
$$

where $\boldsymbol{\Delta}_{2}\left(\left|\xi_{n}-\xi_{*}\right|^{2}\right)=(\widetilde{\mathbf{J}}-\mathbf{J})\left(\xi_{\mathbf{n}}-\xi_{*}\right)$ is the error introduced in replacing $\widetilde{\mathbf{J}}$ by $\mathbf{J}$. Secondly, we expand components of $\xi_{*}$ about $\boldsymbol{\epsilon}=\mathbf{0}$ and write

$$
\xi_{*}=\mathbf{M} \epsilon+\Delta_{3}\left(|\epsilon|^{2}\right)
$$

where $\mathbf{M}=\left(\partial \xi_{*} / \partial \boldsymbol{\epsilon}\right)_{\epsilon=\mathbf{0}}$ is an $N \times N$ matrix, and vector $\boldsymbol{\Delta}_{3}\left(|\epsilon|^{2}\right)$ is the remainder in the expansion. Eliminate $\xi_{*}$ and $\xi_{n+1}$ in Eqs. (2), (4) and (5), and we have

$$
\begin{aligned}
(\mathbf{J}-\mathbf{I}) \mathbf{M} \boldsymbol{\epsilon}= & (\mathbf{J}-k \mathbf{I}) \xi_{n}+\boldsymbol{\Delta}_{1}\left(\left|\xi_{n}-\xi_{*}\right|^{2}\right) \\
& +\boldsymbol{\Delta}_{2}\left(\left|\xi_{n}-\xi_{*}\right|^{2}\right)-(\mathbf{J}-\mathbf{I}) \boldsymbol{\Delta}_{3}\left(|\boldsymbol{\epsilon}|^{2}\right),
\end{aligned}
$$

where $\mathbf{I}$ is the $N \times N$ identity matrix. 
Up to the first order in $\left|\xi_{n}-\xi_{*}\right|$ and $|\epsilon|$, i.e., when $\boldsymbol{\Delta}_{1}\left(\left|\xi_{n}-\xi_{*}\right|^{2}\right), \quad \boldsymbol{\Delta}_{2}\left(\left|\xi_{n}-\xi_{*}\right|^{2}\right)$, and $\boldsymbol{\Delta}_{3}\left(|\epsilon|^{2}\right)$ are all neglected, Eq. (6) becomes

$$
\boldsymbol{\epsilon}_{n}=\mathbf{M}^{-1}(\mathbf{J}-\mathbf{I})^{-1}\left(\mathbf{J}-k_{1} \mathbf{I}\right) \xi_{n} .
$$

Here we have assumed matrices $(\mathbf{J}-\mathbf{I})$ and $\mathbf{M}$ are invertible, redenoted $\epsilon$ by $\epsilon_{n}$ to indicate that the parameter adjustment is for the $n$th iteration of map (1), and redenoted $k$ by $k_{1}$ to indicate the result is up to the first order in $\left|\xi_{n}-\xi_{*}\right|$ and $|\epsilon|$. Equation (7) is the expression of the parameter adjustment in the straight-line stabilization.

Obviously, the parameter adjustment given by Eq. (7) can stabilize the unstable orbit only if the expansion remainders, $\boldsymbol{\Delta}_{1}\left(\left|\xi_{n}-\xi_{*}\right|^{2}\right), \Delta_{2}\left(\left|\xi_{n}-\xi_{*}\right|^{2}\right.$, and $\boldsymbol{\Delta}_{3}\left(|\epsilon|^{2}\right)$, are all negligible in comparison with the linear terms. This means that, from Eq. (6), the stabilization method is valid only if the following condition is satisfied:

$$
\begin{aligned}
\left|\xi_{n}\right|> & \frac{1}{1-\left|k_{1}\right|} \mid \boldsymbol{\Delta}_{1}\left(\left|\xi_{n}-\xi_{*}\right|^{2}\right) \\
& +\boldsymbol{\Delta}_{2}\left(\left|\xi_{n}-\xi_{*}\right|^{2}\right)-(\mathbf{J}-\mathbf{I}) \boldsymbol{\Delta}_{3}\left(|\boldsymbol{\epsilon}|^{2}\right) \mid .
\end{aligned}
$$

Here we have assumed factor $k_{1}$ has the same sign as $k$, based on the fact that $k_{1}$ is an approximation to $k$. Equation (8) is the expression for the size of the stabilization region. Unstable orbits in the region can be stabilized by the straight-line stabilization method, but those outside the region cannot. In the stabilization region, the original system is changed, due to the stabilization process [i.e., due to the change in the linear terms of the map, as given in Eq. (7)], so much that even the stability property is changed. Outside the region, however, effects of the change in the linear terms of the map are suppressed by the (unchanged) nonlinear terms. In this sense, disturbance to the original system outside the region is less than that within the region. The stabilization is global if the stabilization region is the whole $N$-dimensional space, and local if the region is bounded. A local stabilization is desirable when stabilization is needed only in a certain region or when it is required in a practical problem that the change made to the original system for the purpose of stabilization is as small as possible. Equation (8) provides an estimate about how local the stabilization is and how local the change to the original system is. Furthermore the constant $k_{1}$ in Eq. (8) can serve as a means for controlling the size of the stabilization region. Varying $k_{1}$ (but keeping $\left|k_{1}\right|<1$ ), the size of the stabilization region changes and so does the locality of the disturbance to the system.

As an example, consider the following two-dimensional map:

$$
\begin{aligned}
& x_{n+1}=2 x_{n}\left(1-x_{n}\right)+p, \\
& y_{n+1}=2 y_{n}\left(1-x_{n}\right)+q .
\end{aligned}
$$

This map is in form (1) if we let $\xi=(x, y)$ and $\epsilon=(p, q)$. The map has a fixed point near the origin $\xi_{*}=\left(x_{*}, y_{*}\right)$ where $x_{*}=\frac{1}{4}(1-\sqrt{1+8 p})$ and $y_{*}=q /\left(2 x_{*}-1\right)$. When $p$ $=q=0$, the fixed point $\xi_{*}^{0}=(0,0)$ is fully unstable. Matrices $\mathbf{J}$ and $\mathbf{M}$ are both diagonal. Diagonal elements of $\mathbf{J}$ are 2

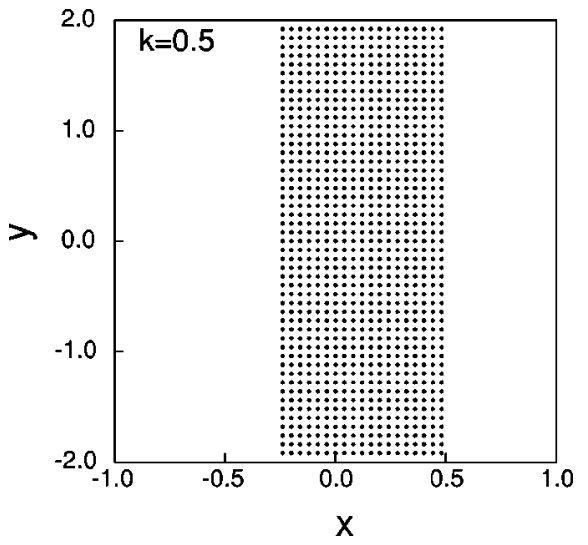

FIG. 1. Numerical results of iterating map (9) with parameters given by Eq. (10) for $k=0.5$. The dotted region is where the straight-line stabilization method works.

and 2, and those of $\mathbf{M}$ are -1 and -1 . According to Eq. (7), the parameters should be adjusted to

$$
p_{n}=(k-2) x_{n}, \quad q_{n}=(k-2) y_{n} .
$$

The size of the stabilization region can be estimated by using Eq. (8). Let $\Delta_{11}$ and $\Delta_{12}$ be the components of vector $\Delta_{1}\left(\left|\xi_{n}-\xi_{*}\right|^{2}\right)$. Up to the second-order terms in $\left|\xi_{n}-\xi_{*}\right|$, they are given by

$$
\begin{aligned}
\Delta_{11}= & \frac{1}{2} \frac{\partial^{2} x_{n+1}}{\partial x_{n}^{2}}\left(x_{n}-x_{*}\right)^{2}+\frac{\partial^{2} x_{n+1}}{\partial x_{n} \partial y_{n}}\left(x_{n}-x_{*}\right)\left(y_{n}-y_{*}\right) \\
& +\frac{1}{2} \frac{\partial^{2} x_{n+1}}{\partial y_{n}^{2}}\left(y_{n}-y_{*}\right)^{2}, \\
\Delta_{12}= & \frac{1}{2} \frac{\partial^{2} y_{n+1}}{\partial x_{n}^{2}}\left(x_{n}-x_{*}\right)^{2}+\frac{\partial^{2} y_{n+1}}{\partial x_{n} \partial y_{n}}\left(x_{n}-x_{*}\right)\left(y_{n}-y_{*}\right) \\
& +\frac{1}{2} \frac{\partial^{2} y_{n+1}}{\partial y_{n}^{2}}\left(y_{n}-y_{*}\right)^{2} .
\end{aligned}
$$

Therefore

$$
\Delta_{1}\left(\left|\xi_{n}-\xi_{*}\right|^{2}\right)=\left(-2\left(x_{n}-x_{*}\right)^{2}, \quad-2\left(x_{n}-x_{*}\right)\left(y_{n}-y_{*}\right)\right) .
$$

It is easy to see that, $\boldsymbol{\Delta}_{2}\left(\left|\xi_{n}-\xi_{*}\right|^{2}\right)=\left(-4 \mathbf{x}_{*}\left(\mathbf{x}_{\mathbf{n}}-\mathbf{x}_{*}\right)\right.$, $\left.-2 \mathbf{y}_{*}\left(\mathbf{x}_{\mathbf{n}}-\mathbf{x}_{*}\right)-2 \mathbf{x}_{*}\left(\mathbf{y}_{\mathbf{n}}-\mathbf{y}_{*}\right)\right)$, up to the second order in $\left|\xi_{n}-\xi_{*}\right|$. Vector $\boldsymbol{\Delta}_{3}\left(\left.|\boldsymbol{\epsilon}|_{*}\right|^{2}\right)$ can be approximated in a way similar to that for $\boldsymbol{\Delta}_{1}\left(\left|\xi_{n}-\xi_{*}\right|^{2}\right)$ and the result is $\boldsymbol{\Delta}_{3}\left(|\epsilon|^{2}\right)$ $=\left(2 p^{2}, 2 p q\right)$. Substituting these results into Eq. (8), we have

$$
\left|x_{n}\right|<\frac{1}{2} \sqrt{1-\left|k_{1}\right|}
$$

This is the estimate to the region of stabilization. Figure 1 shows numerical results for the example. The origin is the fully unstable fixed point of the original map, i.e., map (9) with $p=q=0$. A point in the dotted region goes to the origin under iterations of the map with the control parameters given by Eq. (7). The region is given by $-0.25<x<0.5$ in the 
figure, while the theoretical estimate given by Eq. (8) is

$-0.35<x<0.35$. We consider the agreement acceptable.

\section{PARAMETER ADJUSTMENTS FOR TWO- DIMENSIONAL MAPS}

For two-dimensional $(2 d)$ maps, dependence of the parameter adjustment on the eigenvalues of matrices $\mathbf{J}$ and $\mathbf{M}$ can be explicitly written down. As a $2 \times 2$ matrix, the Jacobian matrix $\mathbf{J}$ for a $2 d$ map has the following three typical forms:

$$
\begin{gathered}
\mathbf{J}_{1}=\left(\begin{array}{cc}
\mu_{1} & 0 \\
0 & \mu_{2}
\end{array}\right), \quad \mathbf{J}_{2}=\mu\left(\begin{array}{cc}
\cos \alpha & -\sin \alpha \\
\sin \alpha & \cos \alpha
\end{array}\right), \\
\mathbf{J}_{3}=\left(\begin{array}{cc}
\mu_{0} & 1 \\
0 & \mu_{0}
\end{array}\right),
\end{gathered}
$$

where $\mu, \mu_{0}, \mu_{1}, \mu_{2}$, and $\alpha$ are all real, and $0 \leqslant \alpha<2 \pi$. If matrix $\mathbf{J}$ is not in one of these forms, it can be transformed into one of them by a coordinate change for $\xi$. Similarly, the typical forms of matrix $\mathbf{M}$ are

$$
\begin{gathered}
\mathbf{M}_{1}=\left(\begin{array}{cc}
\nu_{1} & 0 \\
0 & \nu_{2}
\end{array}\right), \quad \mathbf{M}_{2}=\nu\left(\begin{array}{cc}
\cos \beta & -\sin \beta \\
\sin \beta & \cos \beta
\end{array}\right), \\
\mathbf{M}_{3}=\left(\begin{array}{cc}
\nu_{0} & 1 \\
0 & \nu_{0}
\end{array}\right),
\end{gathered}
$$

where $\nu, \nu_{0}, \nu_{1}, \nu_{2}$, and $\beta$ are all real, and $0 \leqslant \beta<2 \pi$. Therefore Eq. (7) for a planar map $T: \xi_{n} \rightarrow \xi_{n+1}$, where $\xi$ $=(x, y)$ and $\epsilon=(p, q)$, can be written separately for the following nine cases: If $\mathbf{J}=\mathbf{J}_{1}$ and $\mathbf{M}=\mathbf{M}_{1}$,

$$
\begin{aligned}
& p_{n}=\frac{1}{\nu_{1}} a_{1} x_{n}, \\
& q_{n}=\frac{1}{\nu_{2}} a_{2} y_{n} ;
\end{aligned}
$$

if $\mathbf{J}=\mathbf{J}_{1}$ and $\mathbf{M}=\mathbf{M}_{2}$,

$$
\begin{gathered}
p_{n}=\frac{1}{\nu}\left(a_{1} x_{n} \cos \beta+a_{2} y_{n} \sin \beta\right), \\
q_{n}=\frac{1}{\nu}\left(-a_{1} x_{n} \sin \beta+a_{2} y_{n} \cos \beta\right) ;
\end{gathered}
$$

if $\mathbf{J}=\mathbf{J}_{1}$ and $\mathbf{M}=\mathbf{M}_{3}$,

$$
\begin{gathered}
p_{n}=\frac{1}{\nu_{0}}\left(a_{1} x_{n}-\frac{a_{2}}{\nu_{0}} y_{n}\right), \\
q_{n}=\frac{1}{\nu_{0}} a_{2} y_{n} ;
\end{gathered}
$$

if $\mathbf{J}=\mathbf{J}_{2}$ and $\mathbf{M}=\mathbf{M}_{1}$,

$$
p_{n}=\frac{1}{\nu_{1}}\left(b_{1} x_{n}+b_{2} y_{n}\right),
$$

$$
q_{n}=\frac{1}{\nu_{2}}\left(b_{1} y_{n}-b_{2} x_{n}\right) ;
$$

if $\mathbf{J}=\mathbf{J}_{2}$ and $\mathbf{M}=\mathbf{M}_{2}$,

$$
\begin{aligned}
& p_{n}=\frac{1}{\nu}\left[\left(b_{1} x_{n}+b_{2} y_{n}\right) \cos \beta+\left(b_{1} y_{n}-b_{2} x_{n}\right) \sin \beta\right], \\
& q_{n}=\frac{1}{\nu}\left[-\left(b_{1} x_{n}+b_{2} y_{n}\right) \sin \beta+\left(b_{1} y_{n}-b_{2} x_{n}\right) \cos \beta\right] ;
\end{aligned}
$$

if $\mathbf{J}=\mathbf{J}_{2}$ and $\mathbf{M}=\mathbf{M}_{3}$,

$$
\begin{gathered}
p_{n}=\frac{1}{\nu_{0}}\left[\left(b_{1}+\frac{b_{2}}{\nu_{0}}\right) x_{n}+\left(b_{2}-\frac{b_{1}}{\nu_{0}}\right) y_{n}\right], \\
q_{n}=\frac{1}{\nu_{0}}\left(b_{1} y_{n}-b_{2} x_{n}\right) ;
\end{gathered}
$$

if $\mathbf{J}=\mathbf{J}_{3}$ and $\mathbf{M}=\mathbf{M}_{1}$,

$$
\begin{gathered}
p_{n}=\frac{1}{\nu_{1}}\left(a_{0} x_{n}-b_{0} y_{n}\right), \\
q_{n}=\frac{1}{\nu_{2}} a_{0} y_{n} ;
\end{gathered}
$$

if $\mathbf{J}=\mathbf{J}_{3}$ and $\mathbf{M}=\mathbf{M}_{2}$,

$$
\begin{aligned}
& p_{n}=\frac{1}{\nu}\left[\left(a_{0} x_{n}-b_{0} y_{n}\right) \cos \beta+a_{0} y_{n} \sin \beta\right], \\
& q_{n}=\frac{1}{\nu}\left[\left(b_{0} y_{n}-a_{0} x_{n}\right) \sin \beta-a_{0} y_{n} \cos \beta\right] ;
\end{aligned}
$$

if $\mathbf{J}=\mathbf{J}_{3}$ and $\mathbf{M}=\mathbf{M}_{3}$,

$$
p_{n}=\frac{1}{\nu_{0}}\left(a_{0} x_{n}-b_{0} y_{n}-\frac{1}{\nu_{0}} a_{0} y_{n}\right) \text {, }
$$

$$
q_{n}=\frac{1}{\nu_{0}} a_{0} y_{n}
$$

where $\quad a_{i}=\left(\mu_{i}-k\right) /\left(\mu_{i}-1\right), \quad i=0,1,2, \quad b_{0}=(1-k)\left(\mu_{0}\right.$ $-1)^{2}, \quad b_{1}=[k(1-\mu \cos \alpha)+\mu(\mu-\cos \alpha)] /(1-2 \mu \cos \alpha$ $\left.+\mu^{2}\right)$ and $b_{2}=\mu(1-k) \sin \alpha /\left(1-2 \mu \cos \alpha+\mu^{2}\right)$.

\section{STABILIZATION OF FLOWS}

Flow stabilization can be investigated in the following two ways. The first is to introduce a Poincare map for the flow and then study the stabilization problem of the map. The second is to stabilize the flow itself directly, without introducing a Poincare map. We illustrate the two ways by the following example flow on the plane:

$$
\begin{gathered}
\dot{r}=-r(1-r)+p, \quad,|p|<\frac{1}{4}, \\
\dot{\theta}=1,
\end{gathered}
$$


where $(r, \theta)$ are the polar coordinates and $p$ is the control parameter. Obviously, $r=1$ is a limit cycle of the flow with $p=0$, which is unstable because $\left.(d / d r)[-r(1-r)]\right|_{r=1}=1$ $>0$. We want to stabilize a flow not on the cycle and therefore leaving the cycle.

First, we stabilize the flow via a Poincaré map, at $\theta=0$, say. Let initial conditions be $r(t=0)=r_{0}\left(r_{0}\right.$ is a positive real constant) and $\theta(t=0)=0$. To use the results obtained in the previous sections, the fixed point of the map corresponding to the limit cycle $r=1$ should be located at the origin. We therefore make the coordinate change $r \rightarrow r^{\prime}=r-1$ and drop the prime for simplicity of notation. By doing so, we have $r_{n}=r(t=2 n \pi)=-\frac{1}{2}-a+2 a\left[1-e^{4 n \pi a}\left(r_{0}+\frac{1}{2}\right.\right.$ $\left.-a) /\left(r_{0}+\frac{1}{2}+a\right)\right]^{-1}$, where $a=\frac{1}{2} \sqrt{1-4 p}$ and $n \geqslant 0$. Thus the Poincare map is given by

$$
r_{n+1}=-\frac{1}{2}-a+2 a\left[1-e^{4 \pi a} \frac{r_{n}+\frac{1}{2}-a}{r_{n}+\frac{1}{2}+a}\right]^{-1} .
$$

For $p=0$, the map is $r_{n+1}=-1+\left[1-e^{2 \pi} r_{n} /\left(r_{n}+1\right)\right]^{-1}$, whose Jacobian matrix evaluated at the fixed point $r_{*}^{0}=0$ is $\mathbf{J}=e^{2 \pi}$. It is easy to find that map (26) has the fixed point $r_{*}=-\frac{1}{2}+a$ and therefore $\mathbf{M}=\left(\partial r_{*} / \partial p\right)_{p=0}=-1$. From Eq. (7), the parameter adjustment required to stabilize the unstable orbit is given by

$$
p_{n}=-\frac{e^{2 \pi}-k}{e^{2 \pi}-1} r_{n} \approx-r_{n} .
$$

This parameter adjustment is to be performed only when the flow passes the line $\theta=0$ and therefore may be difficult to be implemented in practical problems.

The unstable flow can also be stabilized directly, without introducing a Poincare map. To force the unstable flow to be closer to the limit cycle when time goes on, parameter $p$ should be adjusted so that $\dot{r}<0$ when $r>1$ and $\dot{r}>0$ when $r<1$. A simple choice satisfying these requirements is

$$
p=\kappa r(1-r), \quad \text { for } \quad \kappa>1 .
$$

Figure 2 shows two numerical solutions of flow (25), one with control and the other without control, but both starting from the same point $(r, \theta)=(1.00001,0)$. The thin curve is the flow without control $(p=0)$, which leaves the circle of $r=1$ quickly (two revolutions are shown in the figure). The

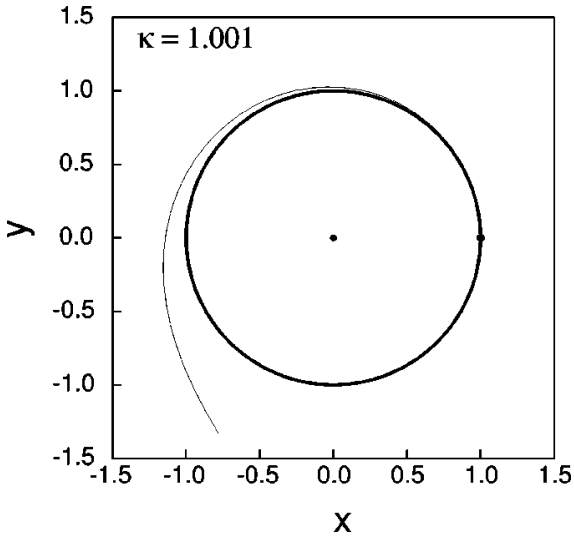

FIG. 2. Stabilization of an unstable flow of Eq. (25). The thin curve is for the unstable flow to be controlled, which leaves the limit cycle of $r=1$. The thick circle is for the controlled flow, which remains near the limit cycle.

thick curve is for the flow with the control parameter given by Eq. (28) and $\kappa=1.001$, which remains near the circle (ten revolutions are shown in the figure).

The stabilization given by the parameter adjustment (28) is global, i.e., the region of stabilization is the whole plane. [The stabilization becomes local if we require, for a given constant $R>1$, the parameter adjustment is given by Eq. (28) for $r \leqslant R$ and zero for $r>R$. In this case, the stabilization region is the disk $r \leqslant R$.] On the other hand, the stabilization for the Poincare map is local because the parameter adjustment given in Eq. (27) is linear in $r_{n}$, and the region of stabilization can be found by using Eq. (8).

\section{CONCLUSIONS}

(i) The region of stabilization for the straight-line stabilization method has been estimated, as given in Eq. (8). The size of the region can be changed by varying the constant $k_{1}$ involved in Eq. (8). (ii) For two-dimensional maps, explicit formulas for the parameter adjustments have been provided for the nine cases. (iii) Unstable flows can be stabilized with or without introducing a Poincaré map.

\section{ACKNOWLEDGMENTS}

This work was partially supported by the Hong Kong Research Grant Council Grant No. HKUST606/95P and by the National Natural Science Foundation of China.
[1] E. Ott, C. Grebogi, and J.A. Yorke, Phys. Rev. Lett. 64, 1196 (1990).

[2] F.J. Romeiras, C. Grebogi, E. Ott, and W.P. Dayawansa, Physica D 58, 165 (1992).

[3] G.V. Osipov, A.K. Kozlov, and V.D. Shalfeev, Phys. Lett. A 247, 119 (1998); G.V. Osipov, L. Glatz, and H. Troger, Chaos, Solitons Fractals 9, 307 (1998).

[4] K. Myneni, T. Barr, N. Corron, and S.D. Pethel, Phys. Rev. Lett. 83, 2175 (1999).
[5] Yang Ling, Liu Zengrong, and Jian-min Mao, Phys. Rev. Lett. 84, 67 (2000).

[6] E.R. Hunt, Phys. Rev. Lett. 67, 1953 (1991); R. Roy, T.W. Murphy, Jr., T.D. Maier, Z. Gills, and E.R. Hunt, ibid. 68, 1259 (1992).

[7] A. Garfinkel, M.L. Spano, W.L. Ditto, and J. Weiss, Science 257, 1230 (1992).

[8] V. Pettov, V. Gaspar, J. Masere, and K. Showalter, Nature (London) 361, 240 (1993). 